\title{
The Prognostic Value of Albumin-to-Alkaline Phosphatase Ratio before Radical Radiotherapy in Patients with Non-metastatic Nasopharyngeal Carcinoma: A Propensity Score Matching Analysis
}

\author{
Jae Sik Kim, MD ${ }^{1}$ \\ Bhumsuk Keam, MD, PhD² \\ Dae Seog Heo, MD, PhD² \\ Doo Hee Han, MD, $P h D^{3}$ \\ Chae-Seo Rhee, MD, $\mathrm{PhD}^{3}$ \\ Ji-hoon Kim, MD, PhD ${ }^{4}$ \\ Kyeong Cheon Jung, MD, $\mathrm{PhD}^{5}$ \\ Hong-Gyun $\mathbf{W u}, \mathrm{MD}, \mathrm{PhD}^{1}$
}

\begin{abstract}
Purpose
We first analyzed the prognostic power of albumin-to-alkaline phosphatase ratio (AAPR) before radical radiotherapy (RT) in non-metastatic nasopharyngeal carcinoma (NPC) patients.
\end{abstract}

\section{Materials and Methods}

The records of 170 patients with biopsy-proven, non-metastatic NPC treated by radical RT between 1998 and 2016 at our institution were retrospectively reviewed. Median followup duration was 50.6 months. All patients received intensity-modulated RT and cisplatin based chemotherapy before, during, or after RT. The major treatment of patients was based on concurrent chemoradiotherapy (92.4\%). The AAPR was calculated by the last value of both albumin and alkaline phosphatase within 1 month immediately preceding RT. The optimal cut-off level of AAPR was determined by using Cutoff Finder, a web-based system. Propensity score matching (PSM) analysis was performed.

\section{Results}

The optimal cut-off level of AAPR was 0.4876. After PSM analysis of whole cohort, an AAPR was not related to survival outcomes. In PSM analysis for patients with locoregionally advanced nasopharyngeal carcinoma (LA-NPC), an AAPR $\geq 0.4876$ was related to better overall survival (OS), progression-free survival (PFS), and locoregional relapse-free survival (LRRFS) (OS: hazard ratio [HR], 0.341; 95\% confidence interval [CI], 0.144 to 0.805; p=0.014; PFS: HR, 0.416; 95\% Cl, 0.189 to 0.914; $p=0.029$; and LRRFS: HR, 0.243; 95\% $\mathrm{Cl}, 0.077$ to $0.769 ; \mathrm{p}=0.016$, respectively).

\section{Conclusion}

The AAPR, inexpensive and readily derived from a routine blood test, could be an independent prognostic factor for patients with LA-NPC. And it might help physicians determine treatment plans by identifying the patient's current status. Future prospective clinical trials to validate its prognostic value are needed.
Correspondence: Hong-Gyun $\mathrm{Wu}, \mathrm{MD}, \mathrm{PhD}$ Department of Radiation Oncology,

Seoul National University College of Medicine, 101 Daehak-ro, Jongno-gu, Seoul 03080, Korea Tel: 82-2-2072-3177

Fax: 82-2-765-3317

E-mail:wuhg@snu.ac.kr

Received November 27, 2018

Accepted January 28, 2019

Published Online January 29, 2019
Key words

Albumin-to-alkaline phosphatase ratio, Nasopharyngeal carcinoma, Prognosis, Radical radiotherapy, Propensity score matching

\section{Introduction}

While nasopharyngeal carcinoma (NPC) may be considered a rare malignancy globally, it has the highest incidence in South-Eastern Asia [1]. The clinical behavior of NPC is also distinct, affecting younger patients with high nodal and dis- tant metastasis [2]. At initial diagnosis, more than $70 \%$ of patients had locoregionally advanced nasopharyngeal carcinoma (LA-NPC) [3]. Currently, the standard treatment of LA-NPC is concurrent chemoradiotherapy (CCRT). With improvement of radiotherapy (RT) technique and chemotherapy regimen, the 5-year overall survival (OS) rate exceeded $80 \%$ [4]. However, NPC recurs in approximately $50 \%$ of 
patients, especially in LA-NPC patients [5,6]. The addition of chemotherapy, such as neoadjuvant (NCT) or adjuvant chemotherapy (ACT), to CCRT has been investigated to improve NPC control. Also, this high recurrence rate emphasizes the need to accurately assess the prognosis of NPC. The American Joint Committee on Cancer (AJCC) TNM staging system is the most important and widely used prognostic factor [7]. However, previous studies showed that sometimes this staging system fails to predict prognosis satisfactorily $[8,9]$. Thus, various serum markers, which can be conveniently obtained, have been developed to predict prognosis [10-16], and defining novel prognostic factor is still of great importance, especially in LA-NPC.

The albumin-to-alkaline phosphatase ratio (AAPR) was reported first as a novel prognostic factor in hepatocellular carcinoma patients [17]. In metastatic NPC patients, low AAPR was associated with poor survival and the authors of that study concluded that the AAPR might be a novel prognostic factor in metastatic NPC patients [18]. Also, the role of ratio between albumin (ALB) and alkaline phosphatase (ALP) as a prognostic factor has been reported in patients with pancreatic ductal adenocarcinoma and advanced hepatocellular carcinoma $[19,20]$. However, to the best of our knowledge, the AAPR has not been studied in non-metastatic NPC patients.

In the current study, we first conducted a retrospective study analyzing the prognostic power of AAPR in nonmetastatic NPC patients. In addition to OS and progressionfree survival (PFS) in the previous study [18], we also examined the relationship between AAPR and other survival outcomes, including locoregional relapse-free survival (LRRFS) and distant metastasis-free survival (DMFS).

\section{Materials and Methods}

\section{Patient selection and evaluation}

We retrospectively reviewed 266 biopsy-proven, nonmetastatic NPC patients who underwent radical RT in Seoul National University Hospital from January 1998 to December 2016. The inclusion criteria were as follows: (1) pathologically proven NPC at our hospital; (2) no previous history of head and neck cancer; (3) no concurrent malignancy; (4) receiving intensity-modulated radiotherapy (IMRT); (5) completion of initially planned RT; (6) receiving chemotherapy before, during, or after RT; and (7) complete baseline laboratory data including serum ALB and ALP levels, within 1 month before RT. Finally, among 266 patients, 170 NPC patients were included in this study.
Medical records of baseline clinical and laboratory data, including pathology, computed tomography (CT) or magnetic resonance imaging (MRI) of the head and neck, systemic work-up by chest CT or positron emission tomography / computed tomography (PET/CT), serum ALB and ALP were retrieved and reviewed from the hospital database. All patients were staged according to the seventh edition of the AJCC staging criteria. The AAPR before RT was calculated by dividing the serum ALB level by the ALP level, which were the last values within 1 month immediately preceding RT. During the study period, there was a change in the measuring equipment of ALB and ALP, but there was no change in the reagent. In addition, clinical chemists confirmed that there was no change in the test value by comparing the before and after tests every time there was a change. The optimal cut-off levels of AAPR, ALB, and ALP for assessing LRRFS were determined by using Cutoff Finder, a webbased system designed by Budczies et al. [18,21]. Patients were divided into two groups based on AAPR 0.4876 (AAPR $<0.4876, \mathrm{n}=25$; AAPR $\geq 0.4876, \mathrm{n}=145$ ).

Patients visited outpatient department 2 weeks after completion of RT. Then, there were four outpatient visits at intervals of 1 month, followed by a 1 year visit every 3 months, every 6 months in the next 3 years and annually thereafter. Post-treatment assessment consisted of history taking physical exams, and flexible fiberoptic endoscopy. Imaging modalities including CT or MRI of head and neck, PET/CT and chest $\mathrm{X}$-ray were performed to assess the treatment response and no evidence of disease. One month after RT, head and neck CT or MRI was performed, and PET/CT was performed 3 months later. The treatment response was evaluated according to Response Evaluation Criteria in Solid Tumors ver. 1.1. If required, biopsy of suspicious recurrent site was conducted. OS rate was calculated using survival information of patients from Ministry of the Interior and Safety in Korea.

\section{RT and chemotherapy}

The technique of IMRT has been previously described [22]. The gross total volume (GTV) was defined by primary tumor and metastatic lymph nodes visualized on either CT or MRI. MRI or PET /CT imaging was used in all patients except five, when a radiation oncologist contoured the GTV. The highrisk clinical target volume (CTV) was defined by 3-dimensional auto-expansion of $0.5 \mathrm{~cm}$ to the GTV and modified regarding anatomical architecture. The intermediate-risk CTV included entire nasopharyngeal mucosa, retropharyngeal, and parapharyngeal space, and involved cervical lymph node level with or without one subsequent uninvolved cervical lymph node level, depending on the physician's decision. Also, intermediate-risk CTV encompassed suspicious 
Table 1. Baseline characteristics of nasopharyngeal carcinoma patients

\begin{tabular}{|c|c|c|c|c|c|c|}
\hline \multirow[b]{2}{*}{ Characteristic } & \multicolumn{3}{|c|}{ Before PSM } & \multicolumn{3}{|c|}{ After PSM } \\
\hline & $\begin{array}{c}\text { AAPR }<0.4876 \\
(n=25)\end{array}$ & $\begin{array}{c}\text { AAPR } \geq 0.4876 \\
(n=145)\end{array}$ & p-value & $\begin{array}{c}\text { AAPR }<0.4876 \\
(n=20)\end{array}$ & $\begin{array}{c}\text { AAPR } \geq 0.4876 \\
(n=80)\end{array}$ & p-value \\
\hline Age (yr) & $55(32-75)$ & $49(16-80)$ & 0.022 & $55(34-75)$ & $51(16-73)$ & 0.158 \\
\hline \multicolumn{7}{|l|}{ Sex } \\
\hline Male & $20(80.0)$ & $103(71.0)$ & 0.494 & $17(85.0)$ & $61(76.2)$ & 0.587 \\
\hline Female & $5(20.0)$ & $42(29.0)$ & & $3(15.0)$ & $19(23.8)$ & \\
\hline \multicolumn{7}{|l|}{ ECOG PS } \\
\hline $0-1$ & $21(84.0)$ & $136(93.8)$ & 0.032 & $18(90.0)$ & $74(92.5)$ & 0.747 \\
\hline 2 & $2(8.0)$ & $1(0.7)$ & & 0 & $1(1.2)$ & \\
\hline Unknown & $2(8.0)$ & $8(5.5)$ & & $2(10.0)$ & $5(6.2)$ & \\
\hline \multicolumn{7}{|c|}{ Pathology (WHO) } \\
\hline Type I & $2(8.0)$ & $11(7.6)$ & 0.655 & 0 & $5(6.2)$ & 0.319 \\
\hline Type II & $13(52.0)$ & $89(61.4)$ & & $11(55.0)$ & $50(62.5)$ & \\
\hline Type III & $10(40.0)$ & $45(31.0)$ & & $9(45.0)$ & $25(31.2)$ & \\
\hline \multicolumn{7}{|l|}{ EBV IHC } \\
\hline Positive & $14(56.0)$ & $85(58.6)$ & 0.462 & $13(65.0)$ & $49(61.2)$ & 0.959 \\
\hline Negative & 0 & $7(4.8)$ & & 0 & 0 & \\
\hline Unknown & $11(44.0)$ & $53(36.6)$ & & $7(35.0)$ & $31(38.8)$ & \\
\hline \multicolumn{7}{|l|}{$\mathrm{T}$ category } \\
\hline $\mathrm{T} 1$ & $6(24.0)$ & $45(31.0)$ & 0.002 & $5(25.0)$ & $26(32.5)$ & 0.649 \\
\hline $\mathrm{T} 2$ & 0 & $35(24.1)$ & & 0 & 0 & \\
\hline $\mathrm{T} 3$ & $5(20.0)$ & $32(22.1)$ & & $5(25.0)$ & $23(28.8)$ & \\
\hline $\mathrm{T} 4$ & $14(56.0)$ & $33(22.8)$ & & $10(50.0)$ & $31(38.8)$ & \\
\hline \multicolumn{7}{|l|}{$\mathrm{N}$ category } \\
\hline N0 & $3(12.0)$ & $16(11.0)$ & 0.829 & $3(15.0)$ & $9(11.2)$ & 0.915 \\
\hline N1 & $11(44.0)$ & $51(35.2)$ & & $8(40.0)$ & $30(37.5)$ & \\
\hline N2 & $8(32.0)$ & $55(37.9)$ & & $8(40.0)$ & $34(42.5)$ & \\
\hline N3 & $3(12.0)$ & $23(15.9)$ & & $1(5.0)$ & $7(8.8)$ & \\
\hline \multicolumn{7}{|l|}{ AJCC stage } \\
\hline I & 0 & $3(2.1)$ & 0.224 & 0 & 0 & 0.711 \\
\hline II & $4(16.0)$ & $29(20.0)$ & & $3(15.0)$ & 10 (12.5) & \\
\hline III & $6(24.0)$ & $57(39.3)$ & & $6(30.0)$ & $32(40.0)$ & \\
\hline IV & $15(60.0)$ & $56(38.6)$ & & $11(55.0)$ & $38(47.5)$ & \\
\hline \multicolumn{7}{|l|}{ CCRT } \\
\hline No & $3(12.0)$ & $10(6.9)$ & 0.632 & $2(10.0)$ & $5(6.2)$ & 0.922 \\
\hline Yes & $22(88.0)$ & $135(93.1)$ & & $18(90.0)$ & $75(93.8)$ & \\
\hline \multicolumn{7}{|l|}{ NCT } \\
\hline No & $11(44.0)$ & $84(57.9)$ & 0.281 & $9(45.0)$ & $41(51.2)$ & 0.803 \\
\hline Yes & $14(56.0)$ & $61(42.1)$ & & $11(55.0)$ & $39(48.8)$ & \\
\hline \multicolumn{7}{|l|}{ ACT } \\
\hline No & $21(84.0)$ & $127(87.6)$ & 0.864 & $18(90.0)$ & $72(90.0)$ & 1.000 \\
\hline Yes & $4(16.0)$ & $18(12.4)$ & & $2(10.0)$ & $8(10.0)$ & \\
\hline
\end{tabular}

Values are presented as median (range) or number (\%). PSM, propensity score matching; AAPR, albumin-to-alkaline phosphatase ratio; ECOG PS, Eastern Cooperative Oncology Group performance status; WHO, World Health Organization; EBV, Epstein-Barr virus; IHC, immunohistochemistry; AJCC, American Joint Committee on Cancer; CCRT, concurrent chemoradiotherapy; NCT, neoadjuvant chemotherapy; ACT, adjuvant chemotherapy. 
A

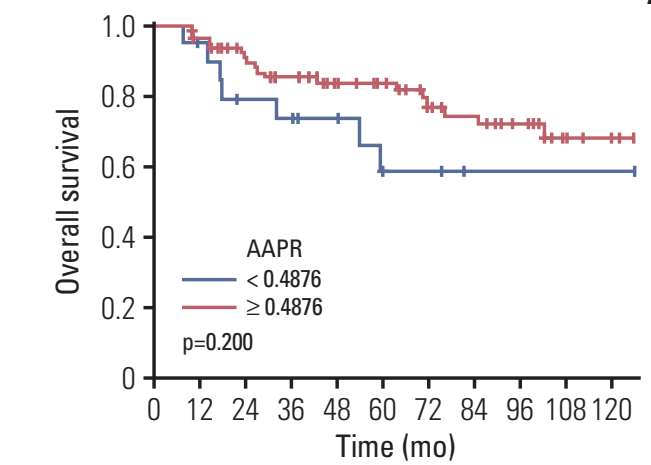

AAPR

$\begin{array}{llllllllllll}0.4876 & 20 & 18 & 14 & 13 & 11 & 8 & 7 & 5 & 5 & 5 & 5\end{array}$

$\begin{array}{llllllllllll}\geq 0.4876 & 80 & 75 & 65 & 59 & 51 & 43 & 32 & 29 & 23 & 15 & 13\end{array}$

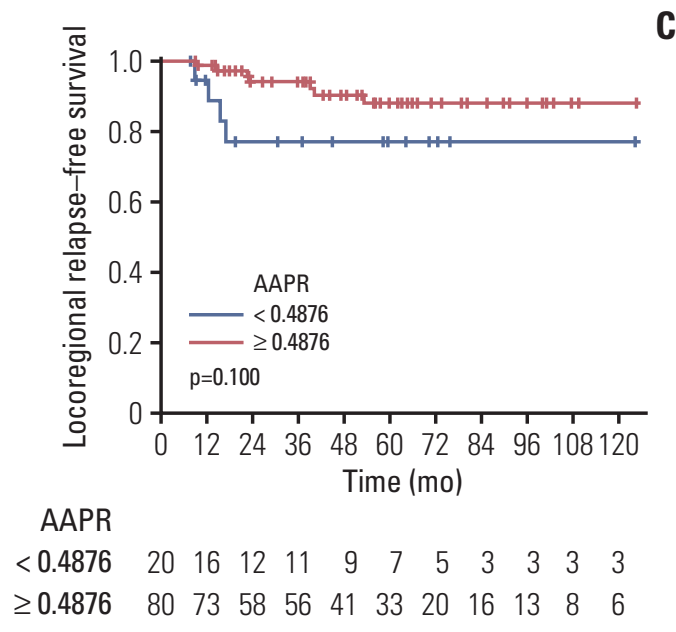

C

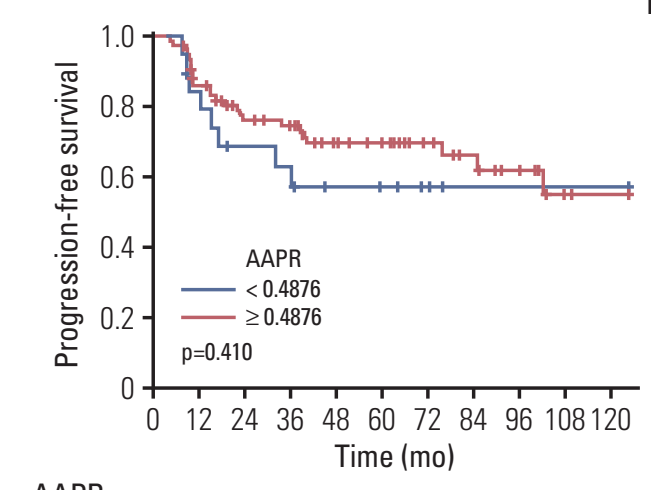

AAPR

$<\begin{array}{lllllllllllll}0.4876 & 20 & 16 & 12 & 11 & 8 & 7 & 5 & 3 & 3 & 3 & 3\end{array}$

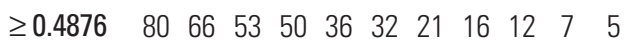

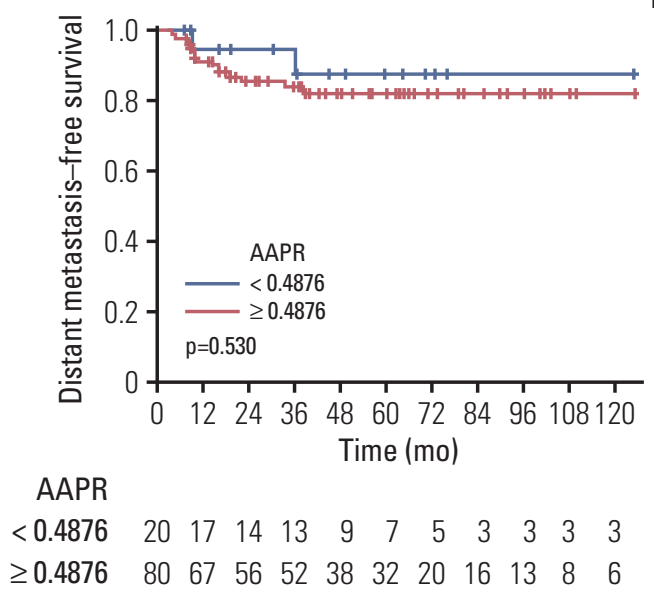

Fig. 1. The Kaplan-Meier survival curves of overall survival (A), progression-free survival (B), locoregional relapse-free survival (C), and distant metastasis-free survival (D) comparing patients with nasopharyngeal carcinoma according to the albumin-to-alkaline phosphatase ratio (AAPR) after propensity score matching.

risk areas among the skull base, inferior sphenoidal sinuses, posterior nasal cavity, posterior maxillary sinuses, and the cavernous sinuses. The low-risk CTV was defined as the cervical lymph nodes which are not included by both high- and intermediate-risk CTV. However, cervical lymph node level IA and IB were not routinely included in any CTVs, if these lymph nodes were not involved. The planning target volumes (PTVs) were defined by 3-dimensional auto-expansion of $0.3 \mathrm{~cm}$ to the CTVs. The total dose of high-, intermediate-, and low-risk PTVs were 63-72 Gy in 2.25-2.4 Gy, 54-63 Gy in 1.8-2.1 Gy, and 44-54 Gy in 1.8-2 Gy, respectively. RT was administrated 5 times per week.

All patients received cisplatin based chemotherapy before, during, or after RT. The major treatment of patients were based on CCRT ( $\mathrm{n}=157,92.4 \%)$. The use of NCT or ACT was determined by the multidisciplinary team. The NCT and ACT were were conducted in 75 and 22 patients, respectively.

\section{Statistical analysis}

OS was defined from the start of any definitive treatment to the date of death from any cause. PFS was defined from the start of any definitive treatment to the date of locoregional failure, distant metastasis, or death from any cause, whichever occurred first. LRRFS and DMFS were defined from the start of any definitive treatment to the date of locoregional failure (LRF) and distant metastasis, respectively.

The chi-square test or Fisher exact test for categorical vari- 
ables and the independent $t$ test for continuous variables were used to compare baseline characteristics. The propensity score matching (PSM) was performed based on age at diagnosis, sex, Eastern Cooperative Oncology Group (ECOG) performance status, immunohistochemistry (IHC) of EpsteinBarr virus (EBV), T category, N category, AJCC stage, and use of NCT, CCRT, and ACT. Propensity score were generated using a multivariable logistic regression model. Then a 1:4 match between the AAPR $<0.4876$ group and $\geq 0.4876$ group was performed using the nearest neighbor-matching method (caliper=0). Survival curves were calculated using the Kaplan-Meier method and were compared using the logrank test. Cox proportional hazards regression was used to identify prognostic factors for survival outcomes. A p-value of $<0.05$ was considered to be statistically significant. All of the statistical analyses were conducted using $\mathrm{R}$ project ver. 3.4.2 (R Foundation for Statistical Computing, Vienna, Austria).

\section{Ethical statement}

This study has been approved by the institutional review board of Seoul National University Hospital (No. H-1705037-852). Considering that this was the retrospective study, the written informed consents of patients were waived.

\section{Results}

\section{Patient characteristics}

The median follow-up duration was 50.6 months. The baseline characteristics were compared between patients according to the pre-RT value of AAPR in Table 1. Although, patients with ECOG performance status 2 were more frequent in the AAPR $<0.4876$ group ( $8.0 \%$ vs. $0.7 \%, \mathrm{p}=0.032)$ and the performance status of 10 patients was unknown, most of the patients had a good performance status (ECOG performance status $0-1$ in $92.4 \%$ of the patients). More than half of patients with an AAPR $<0.4876$ had a T4 category disease $(56.0 \%$ vs. $22.8 \%, \mathrm{p}=0.002)$ but the $\mathrm{N}$ category and AJCC stage were comparable between two groups. The range of ALB level was 34-52 g/ L and the ALP level ranged from 27 to $136 \mathrm{IU} / \mathrm{L}$. Using propensity scores, 20 patients with an AAPR $<0.4876$ were matched successfully with 80 patients with $A A P R \geq 0.4876$ (Table 1 ).

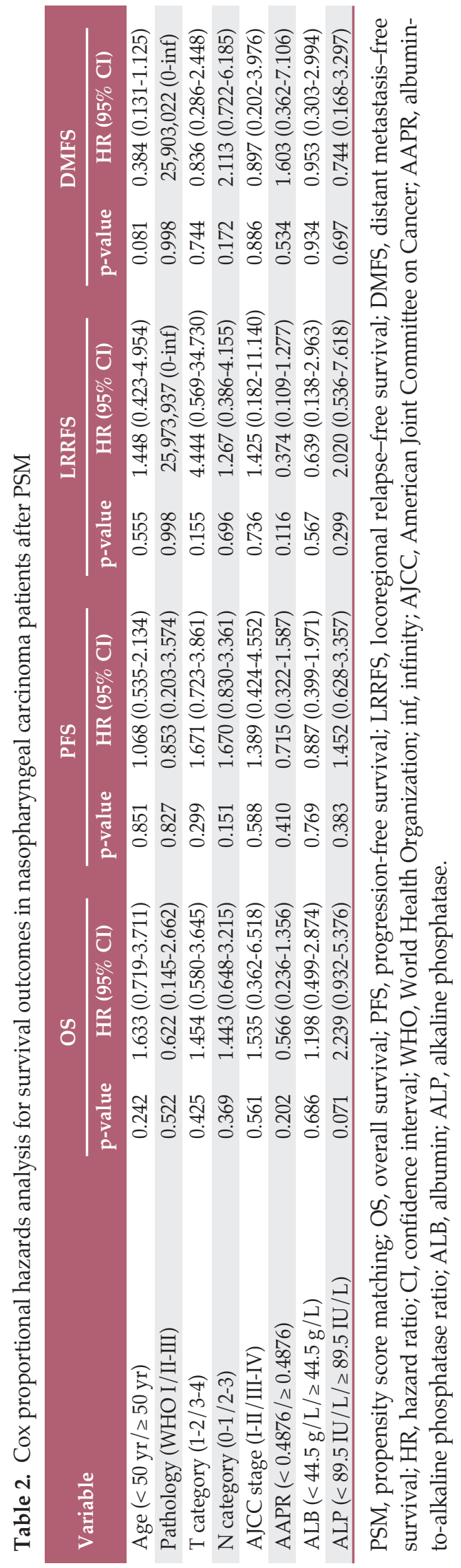


Table 3. Baseline characteristics of locoregionally advanced nasopharyngeal carcinoma patients

\begin{tabular}{|c|c|c|c|c|c|c|}
\hline \multirow[b]{2}{*}{ Characteristic } & \multicolumn{3}{|c|}{ Before PSM } & \multicolumn{3}{|c|}{ After PSM } \\
\hline & $\begin{array}{c}\mathrm{AAPR}<0.4876 \\
(\mathrm{n}=21)\end{array}$ & $\begin{array}{c}\text { AAPR } \geq 0.4876 \\
(n=113)\end{array}$ & p-value & $\begin{array}{c}\text { AAPR }<0.4876 \\
(n=17)\end{array}$ & $\begin{array}{c}\text { AAPR } \geq 0.4876 \\
(n=68)\end{array}$ & p-value \\
\hline Age (yr) & $55(32-75)$ & $49(16-73)$ & 0.023 & $55(34-75)$ & $52.5(21-73)$ & 0.206 \\
\hline \multicolumn{7}{|l|}{ Sex } \\
\hline Male & $16(76.2)$ & $81(71.7)$ & 0.874 & $14(82.4)$ & $53(77.9)$ & 0.947 \\
\hline Female & $5(23.8)$ & $32(28.3)$ & & $3(17.6)$ & $15(22.1)$ & \\
\hline \multicolumn{7}{|l|}{ ECOG PS } \\
\hline $0-1$ & $17(81.0)$ & $106(93.8)$ & 0.034 & $15(88.2)$ & $62(91.2)$ & 0.747 \\
\hline 2 & $2(9.5)$ & $1(0.9)$ & & 0 & $1(1.5)$ & \\
\hline Unknown & $2(9.5)$ & $6(5.3)$ & & $2(11.8)$ & $5(7.4)$ & \\
\hline \multicolumn{7}{|c|}{ Pathology (WHO) } \\
\hline Type I & $2(9.5)$ & $10(8.8)$ & 0.790 & $1(5.9)$ & $5(7.4)$ & 0.888 \\
\hline Type II & $11(52.4)$ & $68(60.2)$ & & $10(58.8)$ & $43(63.2)$ & \\
\hline Type III & $8(38.1)$ & $35(31.0)$ & & $6(35.3)$ & $20(29.4)$ & \\
\hline \multicolumn{7}{|l|}{ EBV IHC } \\
\hline Positive & $12(57.1)$ & $64(56.6)$ & 0.484 & $11(64.7)$ & $41(60.3)$ & 0.956 \\
\hline Negative & 0 & $7(6.2)$ & & 0 & 0 & \\
\hline Unknown & $9(42.9)$ & $42(37.2)$ & & $6(35.3)$ & $27(39.7)$ & \\
\hline \multicolumn{7}{|l|}{$\mathrm{T}$ category } \\
\hline $\mathrm{T} 1$ & $2(9.5)$ & $30(26.5)$ & 0.005 & $2(11.8)$ & $15(22.1)$ & 0.490 \\
\hline $\mathrm{T} 2$ & 0 & $18(15.9)$ & & 0 & 0 & \\
\hline $\mathrm{T} 3$ & $5(23.8)$ & $32(28.3)$ & & $5(29.4)$ & $23(33.8)$ & \\
\hline $\mathrm{T} 4$ & $14(66.7)$ & $33(29.2)$ & & $10(58.8)$ & $30(44.1)$ & \\
\hline \multicolumn{7}{|l|}{$\mathrm{N}$ category } \\
\hline No & $3(14.3)$ & $10(8.8)$ & 0.526 & $3(17.6)$ & $9(13.2)$ & 0.894 \\
\hline N1 & $7(33.3)$ & $25(22.1)$ & & $6(35.3)$ & $21(30.9)$ & \\
\hline N2 & $8(38.1)$ & $55(48.7)$ & & $7(41.2)$ & $31(45.6)$ & \\
\hline N3 & $3(14.3)$ & $23(20.4)$ & & $1(5.9)$ & $7(10.3)$ & \\
\hline \multicolumn{7}{|l|}{ AJCC stage } \\
\hline III & $6(28.6)$ & $57(50.4)$ & 0.108 & $6(35.3)$ & 31 (45.6) & 0.623 \\
\hline IV & $15(71.4)$ & $56(49.6)$ & & $11(64.7)$ & $37(54.4)$ & \\
\hline \multicolumn{7}{|l|}{ CCRT } \\
\hline No & $3(14.3)$ & $8(7.1)$ & 0.502 & $2(11.8)$ & $5(7.4)$ & 0.921 \\
\hline Yes & $18(85.7)$ & 105 (92.9) & & $15(88.2)$ & $63(92.6)$ & \\
\hline \multicolumn{7}{|l|}{ NCT } \\
\hline No & $9(42.9)$ & $59(52.2)$ & 0.582 & $8(47.1)$ & 32 (47.1) & 1.000 \\
\hline Yes & $12(57.1)$ & $54(47.8)$ & & $9(52.9)$ & $36(52.9)$ & \\
\hline \multicolumn{7}{|l|}{ ACT } \\
\hline No & $19(90.5)$ & $98(86.7)$ & 0.907 & $15(88.2)$ & $61(89.7)$ & 1.000 \\
\hline Yes & $2(9.5)$ & $15(13.3)$ & & $2(11.8)$ & $7(10.3)$ & \\
\hline
\end{tabular}

Values are presented as median (range) or number (\%). PSM, propensity score matching; AAPR, albumin-to-alkaline phosphatase ratio; ECOG PS, Eastern Cooperative Oncology Group performance status; WHO, World Health Organization; EBV, Epstein-Barr virus; IHC, immunohistochemistry; AJCC, American Joint Committee on Cancer; CCRT, concurrent chemoradiotherapy; $\mathrm{NCT}$, neoadjuvant chemotherapy; $\mathrm{ACT}$, adjuvant chemotherapy. 
A

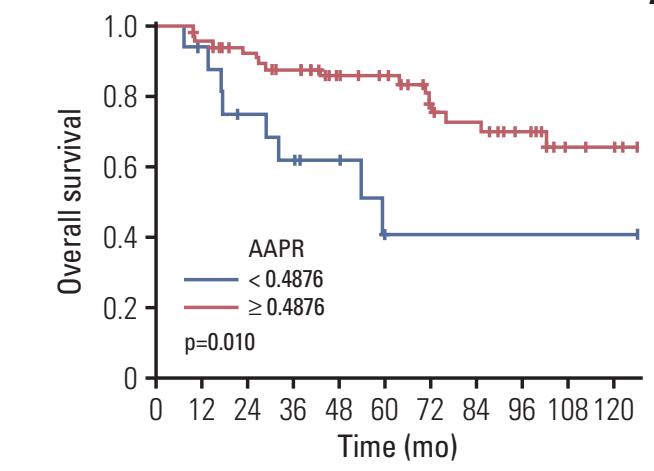

AAPR

$<\begin{array}{lllllllllllll}0.4876 & 17 & 15 & 11 & 9 & 7 & 4 & 3 & 3 & 3 & 3 & 3\end{array}$

$\begin{array}{llllllllllll}20.4876 & 68 & 64 & 57 & 52 & 45 & 39 & 29 & 26 & 20 & 12 & 11\end{array}$

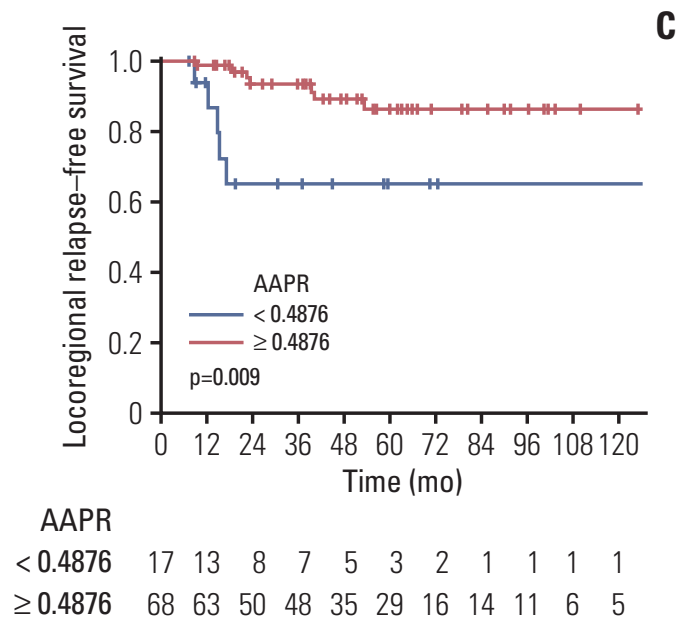

C
B

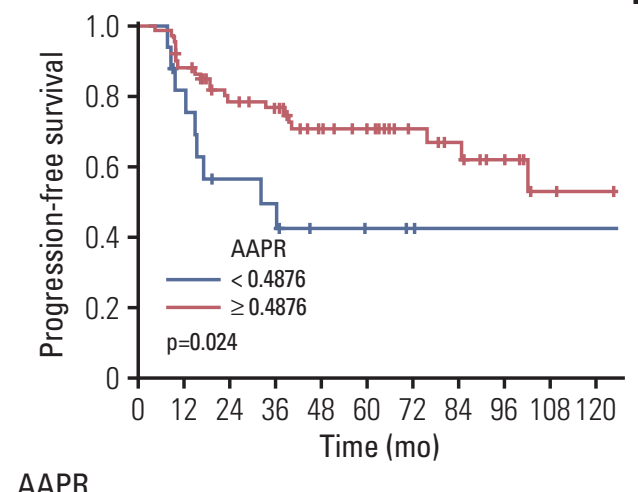

AAPR

$<\begin{array}{llllllllllll}0.4876 & 17 & 13 & 8 & 7 & 4 & 3 & 2 & 1 & 1 & 1 & 1\end{array}$

$\begin{array}{llllllllllll}2.4876 & 68 & 59 & 47 & 44 & 31 & 28 & 17 & 14 & 10 & 5 & 4\end{array}$

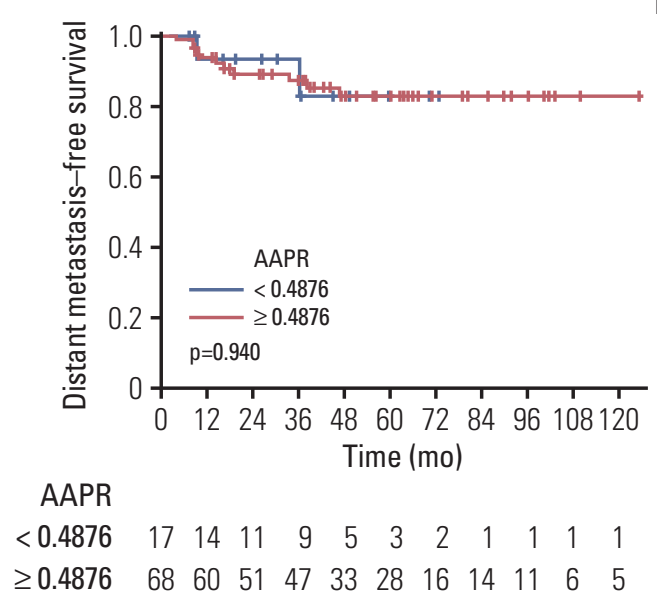

Fig. 2. The Kaplan-Meier survival curves of overall survival (A), progression-free survival (B), locoregional relapse-free survival (C), and distant metastasis-free survival (D) comparing patients with locoregionally advanced nasopharyngeal carcinoma according to the albumin-to-alkaline phosphatase ratio (AAPR) after propensity score matching.

\section{Survival outcomes}

The Kaplan-Meier survival curves are shown in Fig. 1. The 5 -year OS rates in the AAPR $<0.4876$ group and the AAPR $\geq 0.4876$ group were $58.8 \%$ (95\% confidence interval [CI], $38.9 \%$ to $88.8 \%$ ) and $83.7 \%$ (95\% CI, $75.7 \%$ to $92.6 \%$ ), respectively ( $\mathrm{p}=0.200$ ) (Fig. 1A). The 5-year PFS rates in the AAPR $<0.4876$ group and the AAPR $\geq 0.4876$ group were $57.4 \%$ (95\% CI, $38.7 \%$ to $85.0 \%$ ) and $69.9 \%$ (95\% CI, 60.0 to $81.4 \%$ ), respectively ( $\mathrm{p}=0.410)$ (Fig. 1B). Patients with an AAPR $\geq 0.4876$ had higher 5 -year LRRFS rates $(88.1 \%$; $95 \%$ CI, $80.0 \%$ to $97.0 \%$ vs. $77.0 \%$; $95 \%$ CI, $59.5 \%$ to $99.6 \%$ ) but it was not statistically significant ( $\mathrm{p}=0.100$ ) (Fig. 1C). DMFS rates were similar between two groups $(87.2 \%$; $95 \%$ CI, $71.9 \%$ to $100.0 \%$ vs. $81.9 \%$; $95 \%$ CI, $73.4 \%$ to $91.4 \%$, $\mathrm{p}=0.530$ ) (Fig. 1D).
In the Cox proportional hazards analysis for survival outcomes, the AAPR was not a significant prognostic factor (Table 2).

\section{Subgroup analysis of LA-NPC patients}

We conducted subgroup analysis for patients with stage III-IVB NPC. Patient and treatment characteristics of LANPC before and after PSM are shown in Table 3. The KaplanMeier survival curves for LA-NPC patients are shown in Fig. 2. The AAPR $\geq 0.4876$ group in LA-NPC patients had higher 5-year OS, PFS, and LRRFS rates (OS: 85.8\%; 95\% CI, $77.6 \%$ to $94.9 \%$ vs. $41.1 \%$; $95 \%$ CI, $20.6 \%$ to $81.9 \%$; $\mathrm{p}=0.010$; PFS: $71.0 \%$; $95 \%$ CI, $60.4 \%$ to $83.4 \%$ vs. $42.5 \%$; $95 \%$ CI, $23.7 \%$ to $76.4 \%$; $\mathrm{p}=0.024$; and LRRFS: $86.1 \%$; $95 \% \mathrm{CI}, 76.8 \%$ to $96.5 \%$ 


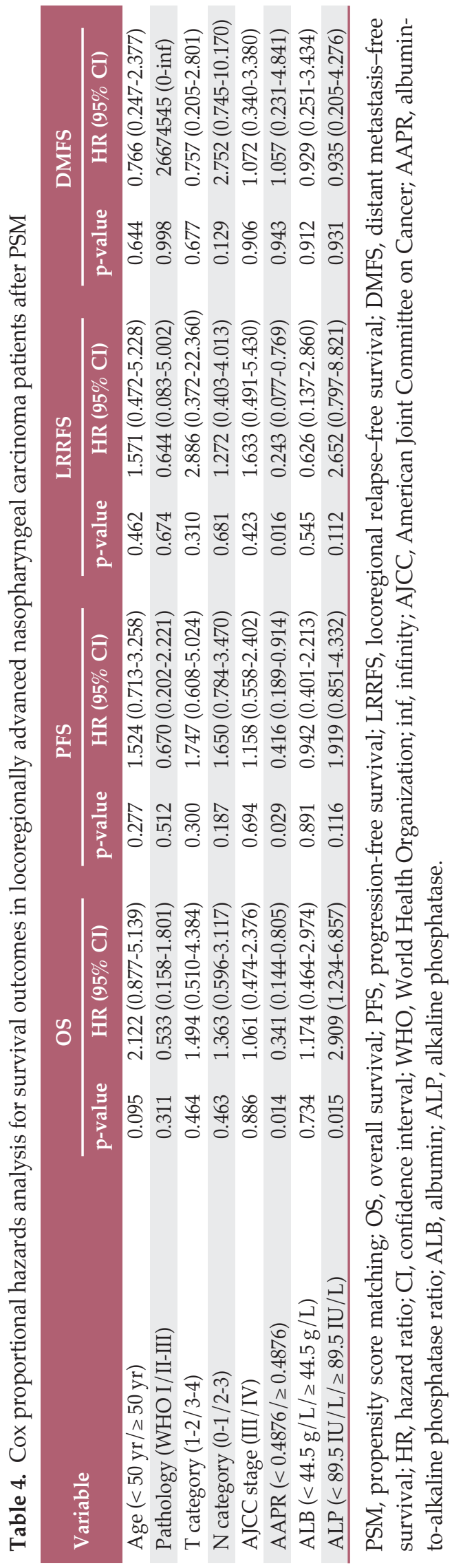

vs. $64.9 \%$; $95 \% \mathrm{CI}, 44.2 \%$ to $95.3 \%$; $\mathrm{p}=0.009)$. There was no significant difference in 5-year DMFS rate between two groups $(83.0 \%$; $95 \%$ CI, $63.5 \%$ to $100.0 \%$ vs. $82.8 \%$; $95 \%$ CI, $73.5 \%$ to $93.3 \%$; $\mathrm{p}=0.940$ ).

In the Cox proportional hazards analysis for LA-NPC patients, high AAPR was a better prognostic factor for OS, PFS and LRRFS (OS: hazard ratio [HR], 0.341; 95\% CI, 0.144 to 0.805; $\mathrm{p}=0.014$; PFS: HR, 0.416 ; $95 \% \mathrm{CI}, 0.189$ to 0.914 ; $\mathrm{p}=0.029$; and LRRFS, HR: $0.243,95 \% \mathrm{CI}, 0.077$ to 0.769 ; $\mathrm{p}=0.016$, respectively) (Table 4 ).

\section{Discussion}

In the present study, we investigated the prognostic value of AAPR before RT in non-metastatic NPC patients. Our results showed that low AAPR was associated with poor OS, PFS, and even LRRFS rates in LA-NPC. These findings suggest that the AAPR is a promising prognostic factor for patients with non-metastatic LA-NPC.

For several decades, there have been efforts to reduce both LRF and side effects of RT in NPC [23-25]. However, there are still significant portion of patients suffering from LRF. A number of prognostic factors of LRF are known, including tumor stage and Epstein-Barr virus status [7,10]. Nonetheless, tailored treatment strategy according to the prognostic factors could not be introduced into daily practice due to lack of clinical experience. Hence, finding prognostic factors other than previously known factors may be helpful in considering individualized treatment. Various studies investigated the role of serum markers due to its easy accessibility [10-16].

Accumulating evidence has clarified the prognostic value of ALB and ALP in various malignancies including NPC, head and neck squamous cell carcinoma and colorectal cancer [11-16]. ALB is considered as a marker of malnutrition and has ability to stabilize DNA during cell cycle [26]. It was known that pretreatment ALB $\leq 43.0 \mathrm{~g} / \mathrm{L}$ in NPC patients was related to poorer OS and DMFS [14]. In terms of malnutrition, low ALB level may reflect profound impairment of immunity, eventually leading to a decreased response to anti-cancer treatment [27]. ALP, an enzyme that dephosphorylates various molecules, such as nucleotides, proteins, and alkaloids [28], is an emerging prognostic factor in several types of carcinomas $[13,15,16]$. Li et al. [15] reported that increased pretreatment ALP level in patients with nonmetastatic NPC receiving radical RT had poor effects on both OS and local recurrence-free survival. However, multivariate analysis did not identify pretreatment ALP level as an independent prognostic factor. The authors suggested that increased ALP level may be associated with severe invasion 
of skull base. Xie et al. [16] also reported that elevated pretreatment ALP level was independent predictor of poor OS and tumor-free survival in patients with non-metastatic NPC. In that study, the authors also explained these findings as a result of bony structure invasion. In addition, they suggested another possibility that ALP may affect the survival of NPC patients through matrix metalloproteinase-9, which is associated with chemoresistance in metastatic gastric carcinoma [29]. The concept of AAPR was first introduced for patients who underwent curative surgery for hepatocellular carcinoma [17], and Nie et al. [18] applied it to metastatic NPC patients. We hypothesized that the AAPR would predict survival outcomes in patients with non-metastatic NPC as well as metastatic NPC.

To our knowledge, this is the first study to examine the prognostic value of AAPR before RT in patients with nonmetastatic NPC. We performed the PSM to control the imbalance between two groups. The Cox proportional hazards analysis showed that an AAPR $<0.4876$ was not associated with poor survival outcomes. We performed subgroup analysis for patients with stage III-IVB NPC, which were considered as LA-NPC, because these patients were expected to have more tumor burden. The AAPR was significantly associated with OS, PFS, and LRRFS in subgroup analysis.

In addition to ALB and ALP, liver function was evaluated with total bilirubin, aspartate aminotransferase, and alanine aminotransferase levels for LA-NPC patients. There were no significant differences in the proportion of patients out of normal range between the two groups. In terms of renal function, there was also no difference between the two groups in patients with glomerular filtration rate less than $60 \mathrm{~mL} / \mathrm{min}$ / $1.73 \mathrm{~m}^{2}$.

As oropharynx infiltration occurred, swallowing became difficult and caused dietary problems, affecting ALB levels. But there was no one who had dietary problems and no difference in oropharynx infiltration ratio between two groups in LA-NPC (low AAPR, 9 patients [52.9\%] vs. high AAPR, 28 [41.2\%]; $\mathrm{p}=0.547)$.

The question of whether the AAPR reflected the patient's ability to perform CCRT and this might result in treatment outcomes could be raised. However, in LA-NPC patients after PSM, only two patients in high AAPR group received concurrent cisplatin with $25 \%$ dose reduction (1 patient due to emesis and the other due to grade 4 neutropenia). ACT was performed in two patients in low AAPR group and seven in high AAPR group. Among them, one patient with low AAPR discontinued chemotherapy because of selfrefusal. One patient with high AAPR experienced progression of disease and stopped chemotherapy. Based on these findings, it could be seen that the AAPR was not related the patient's performance of treatment. The complete remission of primary site in head and neck CT or MRI taken median
1.2 months after RT was observed in two patients (13.3\%) in low AAPR and 28 patients $(41.2 \%)$ in high AAPR group. Although there was a marginal significance $(p=0.072)$, the difference in complete remission rate between the two groups seemed to be the difference of prognosis after treatment. No one received any surgical intervention after CCRT.

Our findings suggested that low AAPR before RT may be associated with poor prognosis in patients with NPC, especially LA-NPC. In particular, the AAPR before RT could predict the risk of LRF, which might be considered for planning of RT. In other words, caution should be taken to de-intensify locoregional treatment for patients with low AAPR. The AAPR might comprehensively reflect the patient's condition such as nutrition and immunity and the characteristics of NPC including severity and treatment resistance that each component of the AAPR implies. That is, low AAPR can be interpreted as malnutrition, decreased immunity, increased treatment resistance, and relatively severe NPC condition. Intervention of ALB for patients with low AAPR might be considered to increase AAPR and correct malnutrition, resulting in better response of treatment.

Tumor stage was not statistically different but showed poor prognosis in the advanced stage. The reason for not having statistical significance might be that the number of patients was small and the distribution of stage was not even. In addition, we thought that the point that the treatment method according to the stage did not differ greatly in the patient group after the PSM was a limit to the statistical verification ability. Our study, unlike previous studies, did not identify ALB and ALP as independent prognostic factors in outcomes other than OS. Therefore, it can be considered that the AAPR predicts the prognosis of LA-NPC better than these factors do.

There were some limitations in our study. First, this study was a retrospective study conducted in a single institution and failed to use an independent cohort confirming the prognostic value of the AAPR. Secondly, we used the most recent AAPR values that we though would be good representations of the patient's condition, but there was a limit because of one measurement. External validation of optimal cutoff of AAPR is also required. Third, selection bias may occur because patients who did not have a routine blood test within 1 month before RT were excluded among patients with NPC who received radical RT between 1998 and 2016. Finally, data on other known biochemical markers such as EBV IHC status and DNA viral load, C-reactive protein, and lactate dehydrogenase, were lacking and were not included in the analysis. Future prospective clinical trials to validate the prognostic value of AAPR are needed and basic research also needs to be conducted to uncover the underlying mechanism.

In conclusion, the AAPR, derived from a routine blood test 
readily and inexpensively, could be an independent prognostic factor for patients with non-metastatic LA-NPC. By obtaining the AAPR before RT, physicians could know the current nutritional status of patients and the characteristics of NPC, which may be helpful in determining the nutritional intervention or treatment plan.

\section{Conflicts of Interest}

Conflict of interest relevant to this article was not reported.

\section{Acknowledgments}

This research was supported by the National R\&D Program for Cancer Control, Ministry of Health and Welfare, Republic of Korea (grant No. 1631200) and the National R\&D Program through the Dongnam Institute of Radiological \& Medical Sciences (DIRAMS) funded by the Ministry of Education, Science and Technology (50595-2018). This research was also supported by a grant from the National Research Foundation of Korea (NRF), which is funded by the Korea government (MEST, Grant No. 2014M2A2A7055063).

\section{References}

1. Torre LA, Bray F, Siegel RL, Ferlay J, Lortet-Tieulent J, Jemal A. Global cancer statistics, 2012. CA Cancer J Clin. 2015;65:87108.

2. Altun M, Fandi A, Dupuis O, Cvitkovic E, Krajina Z, Eschwege F. Undifferentiated nasopharyngeal cancer (UCNT): current diagnostic and therapeutic aspects. Int J Radiat Oncol Biol Phys. 1995;32:859-77.

3. Mao YP, Xie FY, Liu LZ, Sun Y, Li L, Tang LL, et al. Re-evaluation of 6th edition of AJCC staging system for nasopharyngeal carcinoma and proposed improvement based on magnetic resonance imaging. Int J Radiat Oncol Biol Phys. 2009;73: 1326-34.

4. Zhang LN, Gao YH, Lan XW, Tang J, OuYang PY, Xie FY. Effect of taxanes-based induction chemotherapy in locoregionally advanced nasopharyngeal carcinoma: a large scale propensity-matched study. Oral Oncol. 2015;51:950-6.

5. Huncharek M, Kupelnick B. Combined chemoradiation versus radiation therapy alone in locally advanced nasopharyngeal carcinoma: results of a meta-analysis of 1,528 patients from six randomized trials. Am J Clin Oncol. 2002;25:219-23.

6. Teo PM, Chan AT. Treatment strategy and clinical experience. Semin Cancer Biol. 2002;12:497-504.

7. Tang LL, Li WF, Chen L, Sun Y, Chen Y, Liu LZ, et al. Prognostic value and staging categories of anatomic masticator space involvement in nasopharyngeal carcinoma: a study of 924 cases with MR imaging. Radiology. 2010;257:151-7.

8. Higashi K, Ueda Y, Arisaka Y, Sakuma T, Nambu Y, Oguchi $\mathrm{M}$, et al. 18F-FDG uptake as a biologic prognostic factor for recurrence in patients with surgically resected non-small cell lung cancer. J Nucl Med. 2002;43:39-45.

9. Au JS, Law CK, Foo W, Lau WH. In-depth evaluation of the AJCC/UICC 1997 staging system of nasopharyngeal carcinoma: prognostic homogeneity and proposed refinements. Int J Radiat Oncol Biol Phys. 2003;56:413-26.

10. Lo YM, Chan AT, Chan LY, Leung SF, Lam CW, Huang DP, et al. Molecular prognostication of nasopharyngeal carcinoma by quantitative analysis of circulating Epstein-Barr virus DNA. Cancer Res. 2000;60:6878-81.

11. Borda F, Borda A, Jimenez J, Zozaya JM, Prieto C, Gomez M, et al. Predictive value of pre-treatment hypoalbuminemia in prognosis of resected colorectal cancer. Gastroenterol Hepatol. 2014;37:289-95.

12. Lim WS, Roh JL, Kim SB, Choi SH, Nam SY, Kim SY. Pretreatment albumin level predicts survival in head and neck squamous cell carcinoma. Laryngoscope. 2017;127:E437-42.

13. Hung HY, Chen JS, Chien Y, Tang R, Hsieh PS, Wen S, et al. Preoperative alkaline phosphatase elevation was associated with poor survival in colorectal cancer patients. Int J Colorectal Dis. 2017;32:1775-8.

14. Li G, Gao J, Liu ZG, Tao YL, Xu BQ, Tu ZW, et al. Influence of pretreatment ideal body weight percentile and albumin on prognosis of nasopharyngeal carcinoma: long-term outcomes of 512 patients from a single institution. Head Neck. 2014;36: 660-6.

15. Li G, Gao J, Tao YL, Xu BQ, Tu ZW, Liu ZG, et al. Increased pretreatment levels of serum LDH and ALP as poor prognostic factors for nasopharyngeal carcinoma. Chin J Cancer. 2012; 31:197-206.

16. Xie Y, Wei ZB, Duan XW. Prognostic value of pretreatment serum alkaline phosphatase in nasopharyngeal carcinoma. Asian Pac J Cancer Prev. 2014;15:3547-53.

17. Chan AW, Chan SL, Mo FK, Wong GL, Wong VW, Cheung YS, et al. Albumin-to-alkaline phosphatase ratio: a novel prognostic index for hepatocellular carcinoma. Dis Markers. 2015; 2015:564057.

18. Nie M, Sun P, Chen C, Bi X, Wang Y, Yang H, et al. Albuminto-alkaline phosphatase ratio: a novel prognostic index of overall survival in cisplatin-based chemotherapy-treated patients with metastatic nasopharyngeal carcinoma. J Cancer. 2017;8:809-15.

19. Pu N, Gao S, Xu Y, Zhao G, Lv Y, Nuerxiati A, et al. Alkaline phosphatase-to-albumin ratio as a prognostic indicator in pancreatic ductal adenocarcinoma after curative resection. J Cancer. 2017;8:3362-70.

20. Cai X, Chen Z, Chen J, Ma X, Bai M, Wang T, et al. Albuminto-alkaline phosphatase ratio as an independent prognostic factor for overall survival of advanced hepatocellular carcinoma patients without receiving standard anti-cancer thera- 
pies. J Cancer. 2018;9:189-97.

21. Budczies J, Klauschen F, Sinn BV, Gyorffy B, Schmitt WD, Darb-Esfahani S, et al. Cutoff Finder: a comprehensive and straightforward Web application enabling rapid biomarker cutoff optimization. PLoS One. 2012;7:e51862.

22. Wee CW, Keam B, Heo DS, Sung MW, Won TB, Wu HG. Locoregionally advanced nasopharyngeal carcinoma treated with intensity-modulated radiotherapy plus concurrent weekly cisplatin with or without neoadjuvant chemotherapy. Radiat Oncol J. 2015;33:98-108.

23. Tang LL, Tang XR, Li WF, Chen L, Tian L, Lin AH, et al. The feasibility of contralateral lower neck sparing intensity modulation radiated therapy for nasopharyngeal carcinoma patients with unilateral cervical lymph node involvement. Oral Oncol. 2017;69:68-73.

24. Hung TM, Fan KH, Chen EY, Lin CY, Kang CJ, Huang SF, et al. An elective radiation dose of $46 \mathrm{~Gy}$ is feasible in nasopharyngeal carcinoma treated by intensity-modulated radiotherapy: a long-term follow-up result. Medicine (Baltimore). 2017; 96:e6036.

25. Yang H, Chen X, Lin S, Rong J, Yang M, Wen Q, et al. Treat- ment outcomes after reduction of the target volume of intensity-modulated radiotherapy following induction chemotherapy in patients with locoregionally advanced nasopharyngeal carcinoma: a prospective, multi-center, randomized clinical trial. Radiother Oncol. 2018;126:37-42.

26. Arroyo V, Garcia-Martinez R, Salvatella X. Human serum albumin, systemic inflammation, and cirrhosis. J Hepatol. 2014;61:396-407.

27. Chandra RK. Nutrition and immunology: from the clinic to cellular biology and back again. Proc Nutr Soc. 1999;58:681-3.

28. Reiss I, Inderrieden D, Kruse K. Measurement of skeletal specific alkaline phosphatase in disorders of calcium metabolism in childhood. Monatssch Kinderheilkd. 1996;144:885-90.

29. Al-Batran S, Wirtz RM, Pauligk C, Steinmetz K, Probst S, Hartmann JT, et al. Association of elevated matrix metalloproteinase-9 (MMP-9) mRNA expression levels with resistance to chemotherapy and survival in patients with metastatic gastric cancer receiving first-line chemotherapy: results from the FLO versus FLP gastric cancer phase III trial of the AIO. J Clin Oncol. 2008;26(15 Suppl):4544. 